\title{
Photographic observations of Pluto 1991-1994 with the Baldone Schmidt telescope ${ }^{\star}$
}

\author{
V.P. Ryl'kov ${ }^{1}$, A.A. Dement'eva ${ }^{1}$, A. Alksnis ${ }^{2}$ and J.I. Straume ${ }^{2}$ \\ 1 Central Astronomical Observatory of the Russian Academy of Sciences at Pulkovo, Pulkovskoe sh. 65/1, Saint- \\ Petersburg, 196140, Russia \\ e-mail: vryl@pulvz.spb.su \\ 2 Radioastrophysical Observatory, Latvian Academy of Sciences, Turgeneva 19, Riga, LV-1527, Latvia
}

Received June 6; accepted December 21, 1995

\begin{abstract}
Pluto for 1991-1994 are presented. The observations were carried out by Latvian astronomers using the $80 / 120 / 240 \mathrm{~cm}$ Schmidt telescope at Baldone near Riga. 34 plates were reduced by using the 12-21 reference stars from catalogue of southern stars FOCAT-S, created at Pulkovo. The mean reduction errors vary from $0.23^{\prime \prime}$ to $0.34^{\prime \prime}$ for both coordinates. The obtained Pluto positions were compared with the ephemeris JPL DE200. The increase of the mean (O-C) residuals in RA, already noticed in previous works by the Pulkovo authors, is confirmed. In 1994 the $(\mathrm{O}-\mathrm{C})$ residuals with respect DE200 have reached $2.32^{\prime \prime} \pm 0.07^{\prime \prime}$ in $\mathrm{RA}$ and $-0.74^{\prime \prime} \pm 0.07^{\prime \prime}$ in Dec.
\end{abstract}

Key words: astrometry — planets and satellites: individual: Pluto

\section{Introduction}

Schmidt telescopes have more light-gathering power, are faster and permit to reach fainter magnitudes than astrographs with lenses. As a consequence, they are widely used for positional astrometry of faint objects (Steinert 1987; Murray et al. 1986; Andersen 1971). For example, the Schmidt telescopes of the Tautenburg and Bergedorf observatories have been routinely used for astrometry for a long time. The Guide Star Catalogue (GSC) of positions and magnitudes of 19 millions of stars has also been obtained with two Schmidt telescopes (Lasker et al. 1990). Moreover, since 1969 italian astronomers observe the faint planet Pluto with the $67 / 92 \mathrm{~cm}$ Schmidt telescope of the Asiago Astrophysical Observatory and, more recently, with 100/162 $\mathrm{cm}$ Schmidt telescope located at ESO-La Silla (Barbieri et al. 1988). They obtain highly accurate astrometric positions of the planet.

The 80/120/240 cm Schmidt telescope of the Baldone Radioastrophysical Observatory (Observatory code 69) is located in the town of Baldone (near Riga, Latvia) and has been used before for measurements aimed to determining the photometric properties and coordinates with accuracy $1-2^{\prime \prime}$ of newly found carbon stars. Moreover it has been

Send offprint requests to: V.P. Ryl'kov

${ }^{\star}$ Table 2 is also available at the CDS via anonymous ftp 130.79.128.5 used to obtaining of the coordinates of minor planets and comets, in particular during the participation in the International Halley Watch astrometry network (Alksnis et al. 1986). The astrometric accuracy of these positions was better than $0.7^{\prime \prime}$.

In the years 1987-1990, the two Pulkovo authors of this paper (V.P.R. and A.A.D.) have often used the Baldone Schmidt to observe the photometric part of the Pul PM project (Pulkovo stellar proper motion program in selected areas with galaxies) and their attention has been drawn to the good quality of the stellar images on the whole plate. This led to the idea of using the telescope for positional observations of Pluto.

The optical and photometric study of the field of the telescope has been made immediately after its establishment in 1967 and has shown the high quality of the optics (Alksnis 1973). The field of the telescope is circular with a diameter of $4^{\circ} 46^{\prime}$, the plates are $24 \times 24 \mathrm{~cm}$, and are curved in a special plate-holder during exposure. The average scale on the focal plane is $86^{\prime \prime} / \mathrm{mm}$, that is slightly better than the scales of the zone astrographs normally used for photographic positional astrometry.

The main difficulty in using Schmidt telescopes for astrometry is the field curvature wich can be removed either by curving the plate in a special plate-holder or by using the Piazzi-Smith lense. In order to avoid additional light losses and the appearance of coma, the Piazzi-Smith lense 
Table 1. The accuracy of the reduction of the Pluto positions 1991-94 from the plates of the Baldone Schmidt telescope and Pulkovo Normal astrograph

\begin{tabular}{lcccccccccc}
\hline & \multicolumn{3}{c}{ Schmidt } & \multicolumn{3}{c}{ telescope } & \multicolumn{3}{c}{ Normal } & \multicolumn{3}{c}{ astrograph } \\
\hline Year & \multicolumn{3}{c}{$\begin{array}{c}\text { Number } \\
\text { posit. }\end{array}$} & stars & $\begin{array}{c}\text { Mean } \\
S_{x}\end{array}$ & $\begin{array}{c}\text { rms err } \\
S_{y}\end{array}$ & $\begin{array}{c}\text { Number } \\
\text { pl. }\end{array}$ & stars & $\begin{array}{c}\text { Mean } \\
S_{x}\end{array}$ & $\begin{array}{c}\text { rms err } \\
S_{y}\end{array}$ \\
\hline 1991 & 8 & 14 & 14 & $0.281^{\prime \prime}$ & $0.328^{\prime \prime}$ & 9 & $6-10$ & $0.438^{\prime \prime}$ & $0.426^{\prime \prime}$ \\
1992 & 10 & 15 & $13-15$ & 0.230 & 0.275 & 5 & 10 & 0.440 & 0.512 \\
1993 & 9 & 13 & $18-21$ & 0.293 & 0.337 & 7 & 15 & 0.340 & 0.386 \\
1994 & 7 & 13 & $12-14$ & 0.319 & 0.281 & 4 & 7 & 0.216 & 0.242 \\
\hline
\end{tabular}

is seldom used with large telescopes. Usually, bending the plate in its holder decreases the aberration but the astrometry of the photographic field is worsened.

After the exposure the plate returns flat and the $\Delta r$ relative shifts of the images depend on the elastic characteristics of the plate and on the thickness of the glass. The theory of the displacement of images shows that for spherically curved glass plates, this shift is given by the combination of linear and cubic terms and is caused by inclination and radial distortion (Murray 1986).

To obtain the coefficient of the radial cubic distortion $C_{\mathrm{d}}$ for the photographic field $\left(4.3^{\circ} \times 4.3^{\circ}\right)$ of the Schmidt telescope some plates of Pleiades open cluster, obtained in 1967 and 1987 have been analyzed. The average value of the coefficient $C_{\mathrm{ds}}=-5.510^{-8} \mathrm{~mm}^{-2}$ has been computed both graphically and as the distortion coefficient in the calculated reduction polynomials (Ryl'kov 1993).

The differences between the $C_{\mathrm{d}}$ values for the individual plates and the average coefficient $C_{\mathrm{ds}}$ are mainly in the range $0.1-:-0.310^{-8} \mathrm{~mm}^{-2}$ and are probably caused by the slightly different curvature of the plates in the plateholder. According to Ryl'kov (1993), a similar range of the radial cubic distortion coefficient gave the following displacements $(\Delta a, \Delta d)$ in the calculated coordinates for two intervals of stellar distances $R$ from the center of the field:

1. for $0^{\circ} \leq R \leq 1^{\circ}$ :

if $\Delta C=0.210^{-8} \mathrm{~mm}^{-2}$, then $\Delta a=0.001 \mathrm{~s}, \Delta d=0.01^{\prime \prime}$; if $\Delta C=0.510^{-8} \mathrm{~mm}^{-2}$, then $\Delta a=0.002 \mathrm{~s}, \Delta d=0.03^{\prime \prime}$, 2. for $1^{\circ}<R \leq 2^{\circ}$ :

if $\Delta C=0.210^{-8} \mathrm{~mm}^{-2}$, then $\Delta a=0.003^{\mathrm{s}}, \Delta d=0.04^{\prime \prime}$;

if $\Delta C=0.510^{-8} \mathrm{~mm}^{-2}$, then $\Delta a=0.006^{\mathrm{s}}, \Delta d=0.10^{\prime \prime}$.

So, the use of the average distortion coefficient $C_{\mathrm{ds}}$ for the plates taken with the Baldone Schmidt telescope may introduce in the calculated positions an average variation which, even on the border of the field, is not larger than $0.06^{\prime \prime}$. Using this coefficient, the rms errors for coordinate reduction are halved (Ryl'kov 1993) and practically become similar to those estimated for the plates of the Pulkovo Normal astrograph (about $0.2-0.3^{\prime \prime}$ ). Thus, the Baldone Schmidt telescope is usable for astrometric purposes, at least in a radius of $1.5^{\circ}$ from the center of the field, where the application of the average distortion coefficient $C_{\mathrm{ds}}$ guarantees that positions of objects with errors smaller than $0.02^{\prime \prime}$ are obtained.

\section{Observations}

To confirm that the value of $(\mathrm{O}-\mathrm{C})_{\mathrm{RA}}$ after 1990 became greater than $1.5^{\prime \prime}$, which was obtained with Pulkovo Normal astrograph, photographic positional observations of Pluto with the Baldone Schmidt telescope were organized in the spring of 1991 by A. Alksnis. The observations continued during the next three years usually in the AprilMay period, when Pluto is near opposition and bright summer nights have not yet set in at Baldone.

The observations of the year 1991, when 8 plates with 14 images of Pluto have been obtained, were immediately reduced and gave good results, characterized by high internal accuracy and good agreement with the reduction errors $S_{x}, S_{y}$ and the average annual (O-C) residuals of the positions of Pluto obtained with other telescopes (Ryl'kov et al. 1993).

The results of the observations of Pluto with the Baldone Schmidt telescope during 1992-1994 are given in this paper. The observing techniques and the methods used for the mesurement and the analysis of the data have been described in previous paper (Ryl'kov et al. 1993). During the three spring periods 1992-1994, we obtained 26 plates with 41 images of Pluto in short observing runs; in some cases, two or three images were photographed on the same plate.

The emulsion ORWO ZU21 with filter GG13 (photometrically close to $B$ band in the Johnson $U B V$ system) was used, with exposure times in the range 7-9 min in 1991-1993 and up to $12 \mathrm{~min}$ in 1994. The images of the stars and Pluto are good in most plates, although sometimes the second image of Pluto is fainter than the first one. Pluto can be identified and very well measured on all plates. All plates of the years 1992-1994 at Baldone were obtained by A. Alksnis and J.I. Straume and were 
Table 2. Equatorial geocentric coordinates of Pluto from the observations 1991-94 with the Baldone Schmidt telescope

\begin{tabular}{|c|c|c|c|c|c|c|c|c|}
\hline No pl & Date & JD & RA & $\begin{array}{l}\text { FK5, J2000 } \\
\text { h m s }\end{array}$ & $\begin{array}{l}\text { Dec. } \\
0,11\end{array}$ & $\begin{array}{c}(\mathrm{O}-\mathrm{C})_{\mathrm{RA}} \\
\mathrm{s}\end{array}$ & $\underset{\prime \prime}{(\mathrm{O}-\mathrm{C})_{\mathrm{Dec}}}$ & $\mathrm{Obs}^{*}$ \\
\hline & 1991 & $2448 \ldots$ & & & & & & \\
\hline 18835 & May 7 & 384.475058 & 15 & 2343.797 & -22524.27 & 0.158 & 0.29 & St. \\
\hline 18836 & May 7 & 384.485417 & 15 & 2343.725 & -22524.28 & 0.151 & 0.03 & St. \\
\hline \multirow[t]{2}{*}{18837} & May 8 & 385.473900 & 15 & 2337.487 & -22501.60 & 0.146 & -0.22 & Eg. \\
\hline & May 8 & 385.480787 & 15 & 2337.443 & -22501.09 & 0.145 & 0.12 & Eg. \\
\hline \multirow[t]{2}{*}{18842} & May 9 & 386.456944 & 15 & 2331.254 & -22438.90 & 0.124 & 0.17 & St. \\
\hline & May 9 & 386.463542 & 15 & 2331.229 & -22438.23 & 0.140 & 0.69 & St. \\
\hline 18843 & May 9 & 386.473505 & 15 & 2331.172 & -22438.66 & 0.146 & 0.04 & St. \\
\hline 18844 & May 9 & 386.483333 & 15 & 2331.133 & -22439.04 & 0.170 & -0.55 & St. \\
\hline \multirow[t]{3}{*}{18845} & May 10 & 387.459375 & 15 & 2324.944 & -22416.99 & 0.158 & -0.12 & Jr. \\
\hline & May 10 & 387.467708 & 15 & 2324.878 & -22417.40 & 0.145 & -0.71 & Jr. \\
\hline & May 10 & 387.473264 & 15 & 2324.861 & -22415.77 & 0.163 & 0.79 & Jr. \\
\hline \multirow[t]{3}{*}{18847} & May 13 & 390.448900 & 15 & 2305.961 & -22313.97 & 0.135 & 0.00 & $\mathrm{Pu}$. \\
\hline & May 13 & 390.455035 & 15 & 2305.933 & -22313.99 & 0.146 & -0.14 & $\mathrm{Pu}$. \\
\hline & $\begin{array}{c}\text { May } 13 \\
1992\end{array}$ & 390.463831 & 15 & 2305.882 & -22314.13 & 0.151 & -0.46 & $\mathrm{Pu}$. \\
\hline 19089 & Apr 11 & 723.515081 & 15 & 3542.386 & -33837.84 & 0.180 & -0.63 & St. \\
\hline 19090 & Apr 11 & 723.528299 & 15 & 3542.305 & -33837.12 & 0.164 & 0.66 & St. \\
\hline \multirow[t]{2}{*}{19091} & Apr 11 & 723.537465 & 15 & 3542.276 & -33836.85 & 0.180 & -0.35 & St. \\
\hline & Apr 11 & 723.545347 & 15 & 3542.219 & -33836.32 & 0.162 & 0.92 & St. \\
\hline 19105 & Apr 24 & 737.477396 & 15 & 3426.820 & -33135.19 & 0.181 & -0.30 & St. \\
\hline \multirow[t]{2}{*}{19106} & Apr 24 & 737.489606 & 15 & 3426.739 & -33134.84 & 0.171 & -0.30 & St. \\
\hline & Apr 24 & 737.495856 & 15 & 3426.708 & -33134.58 & 0.176 & -0.21 & St. \\
\hline \multirow[t]{2}{*}{19107} & Apr 25 & 737.506840 & 15 & 3426.638 & -33134.41 & 0.171 & -0.35 & St. \\
\hline & Apr 25 & 737.512685 & 15 & 3426.623 & -33133.68 & 0.190 & 0.20 & St. \\
\hline 19108 & Apr 25 & 737.521713 & 15 & 3426.553 & -33133.93 & 0.172 & -0.29 & St. \\
\hline 19140 & May 19 & 762.383137 & 15 & 3151.977 & -32208.73 & 0.168 & -0.12 & St. \\
\hline \multirow[t]{2}{*}{19141} & May 19 & 762.392396 & 15 & 3151.897 & -32208.90 & 0.147 & -0.44 & St. \\
\hline & May 19 & 762.398426 & 15 & 3151.877 & -32208.39 & 0.165 & -0.03 & St. \\
\hline \multirow[t]{2}{*}{19142} & May 19 & 762.407975 & 15 & 3151.788 & -32207.70 & 0.137 & 0.50 & St. \\
\hline & May 19 & 762.413866 & 15 & 3151.751 & -32208.48 & 0.137 & -0.37 & St. \\
\hline
\end{tabular}

reduced at the Main Astronomical Observatory of the Russian Academy of Sciences by V.P. Rylkov and A.A. Dement'eva.

The measurements have been performed with semiautomatic coordinate measuring machine "ASCORECORD". For each plate, a number of reference stars ranging from 12 to 21 were selected from the FOCAT-S catalogue, created at Pulkovo in 1987-1989. All stars had known proper motion and were located in an area of roughly $2 \times 2^{\circ}$ around the center of the plate. These reference stars are those used for the plates of the Normal astrograph obtained during the same period of observations.

The astrometric coordinate reduction for plates obtained with the Schmidt telescope were made by calculating the coefficient of cubic distortion $C_{\mathrm{ds}}$ to compensate the curvature of the field of the straightened plate (Mur- ray 1986). The measured coordinates were corrected for the third degree distortion with the following formulae:

$$
\begin{gathered}
x_{\mathrm{o}}=\left(x-x_{\mathrm{c}}\right)-C_{\mathrm{ds}}\left(x-x_{\mathrm{c}}\right) r^{2} \\
y_{\mathrm{o}}=\left(y-y_{\mathrm{c}}\right)-C_{\mathrm{ds}}\left(y-y_{\mathrm{c}}\right) r^{2} \\
r^{2}=\left(x-x_{\mathrm{c}}\right)^{2}+\left(y-y_{\mathrm{c}}\right)^{2},
\end{gathered}
$$

where $x_{\mathrm{c}}, y_{\mathrm{c}}-$ the coordinates of the center of the field, $C_{\mathrm{ds}}$ - radial cubic distortion coefficient. The coordinates of Pluto were calculated with the personal computer AT386. The programme "PL-EIGHT" computed the distortion with the formulae given above and performed the

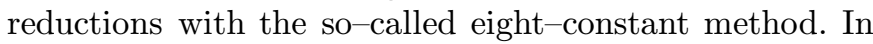
this method, the constants of the equations (see below) which give the relationship between the measured $(x, y)$ coordinates and the tangential $(X, Y)$ coordinates are determined by taking advantage of the linear dependence. 
Table 2. continued

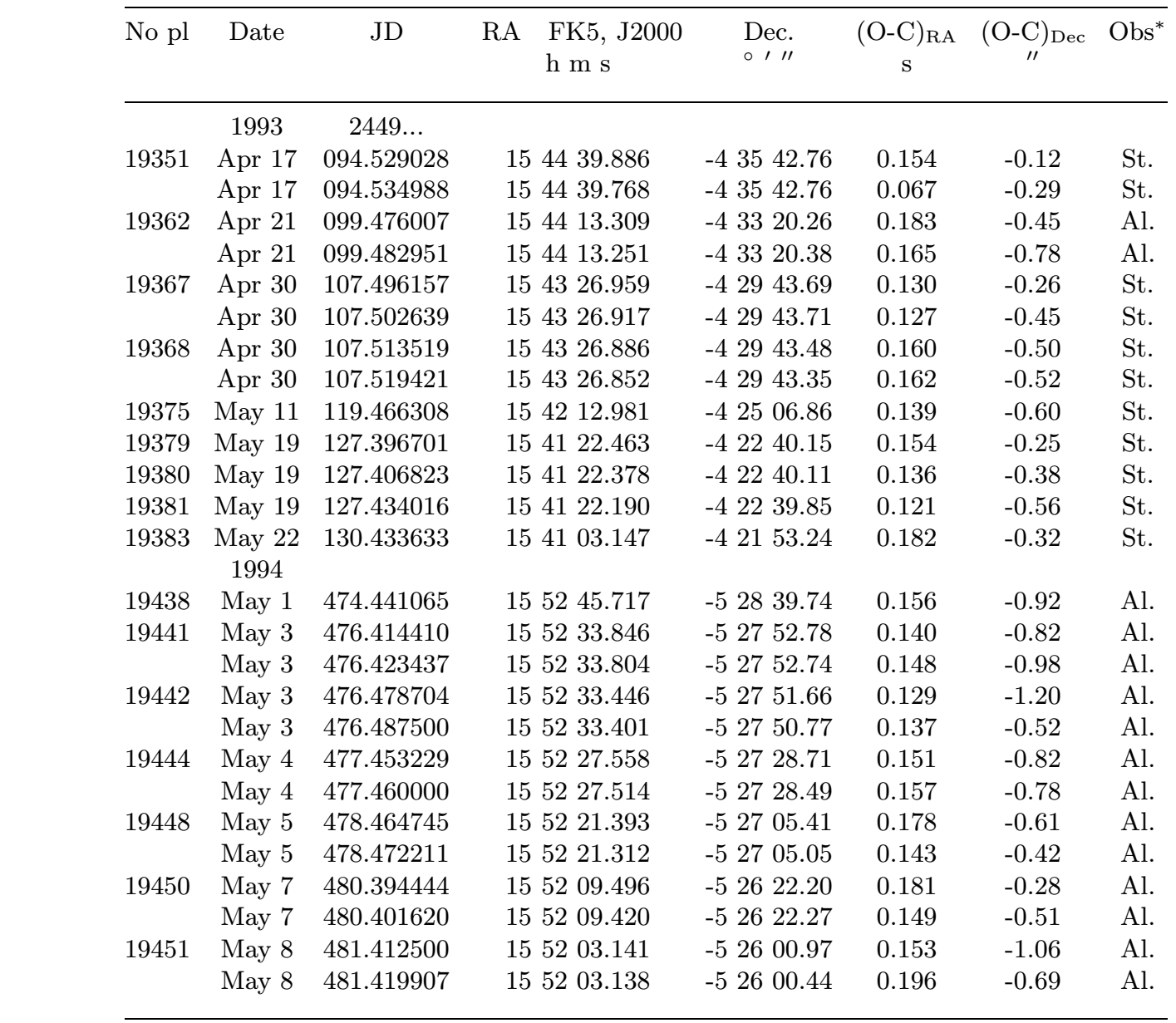

* - Observers with the Baldone Schmidt telescope:

Al. - A. Alksnis, Eg. - I. Eglitis, Jr. - I. Jurgitis, Pu. - I. Pundure, St. - J.I. Straume.

Non-linear terms are also introduced to compensate for the inaccuracy of the assumed position of the optical center of the plate:

$$
\begin{aligned}
& X-x=a_{1} x+b_{1} y+c_{1}+p x^{2}+q x y \\
& Y-y=a_{2} x+b_{2} y+c_{2}+p x y+q y^{2}
\end{aligned}
$$

To compare the results obtained with the Baldone Schmidt telescope with those obtained with the Normal astrograph at Pulkovo, for each of the four years of observation the following data are reported in Table 1: the number of obtained photoplates; the number of images of Pluto (as mentioned above, some Schmidt plates have 2 or 3 exposures); the number of used reference stars and the mean reduction errors $S_{x}, S_{y}$, which are the rms errors and include also the atmospheric and instrumental errors, together with the measurement and catalogue errors of the reference stars.
The individual values of $S_{x}, S_{y}$ for each plate range from $0.15^{\prime \prime}$ to $0.40^{\prime \prime}$ for both coordinates. Their average values for the Schmidt telescope are not greater than $0.34^{\prime \prime}$ and are similar to the typical instrumental errors reported for astrometric telescopes. In Table 1 , the $S_{x^{-}}$and $S_{y}$ values for 1991 are those published by Ryl'kov et al. (1993) and slightly revised because of taking into account the radial cubic distortion. The rather large average values of $S_{x}, S_{y}$ for both telescopes could be explained by worse observing conditions of Pluto (large zenith distance $z>63^{\circ}$ ) in comparison with the previous years.

The general conclusion is: the star positions performed on plates taken both with the Normal astrograph and the Schmidt telescope have the same degree of accuracy and are in good agreement with the accuracy of the reference catalogue.

It is interesting to compare the reduction accuracy of the positions of Pluto obtained from the Baldone Schmidt plates with that of other Schmidt telescopes used for 
Table 3. The mean residuals (O-C) in RA and in DE for positions of Pluto 1991-94 from the plates of the two telescopes (arcsec)

\begin{tabular}{cccccc}
\hline $\begin{array}{c}\text { Year } \\
\text { obs. }\end{array}$ & $\begin{array}{c}\text { Range } \\
(\mathrm{O}-\mathrm{C})_{\mathrm{RA}}\end{array}$ & $\begin{array}{c}\text { Schmidt } \\
(\mathrm{O}-\mathrm{C})_{\mathrm{RA}} \pm \mathrm{Er}\end{array}$ & $\begin{array}{c}\text { telescope } \\
(\mathrm{O}-\mathrm{C})_{\operatorname{Dec}} \pm \mathrm{Er}\end{array}$ & $\begin{array}{c}\text { Normal } \\
(\mathrm{O}-\mathrm{C})_{\mathrm{RA}} \pm \mathrm{Er}\end{array}$ & $\begin{array}{c}\text { astrograph } \\
(\mathrm{O}-\mathrm{C})_{\mathrm{Dec}} \pm \mathrm{Er}\end{array}$ \\
\hline 1991 & $1.80-2.54$ & $2.22 \pm 0.05$ & $-0.01 \pm 0.11$ & $2.10 \pm 0.27$ & $-0.18 \pm 0.24$ \\
1992 & $2.06-2.85$ & $2.50 \pm 0.06$ & $-0.07 \pm 0.12$ & $2.34 \pm 0.14$ & $-0.03 \pm 0.12$ \\
1993 & $1.01-2.74$ & $2.16 \pm 0.13$ & $-0.42 \pm 0.05$ & $2.46 \pm 0.29$ & $-0.22 \pm 0.21$ \\
1994 & $1.95-2.96$ & $2.32 \pm 0.07$ & $-0.74 \pm 0.07$ & $2.59 \pm 0.39$ & $-0.56 \pm 0.24$
\end{tabular}

astrometry, for example with the Schmidt telescope of the Asiago Astrophysical Observatory (the scale $95.8^{\prime \prime} / \mathrm{mm}$, Gemmo \& Barbieri 1994) and the ESO-La Silla Schmidt telescope (the scale $67.5^{\prime \prime} / \mathrm{mm}$ ). The average reduction errors, derived from 30-40 AGK3 reference stars in the period 1979-1987 for the plates of both telescopes were $0.42^{\prime \prime}$ in RA and $0.39^{\prime \prime}$ in Dec. (Barbieri et al. 1988). The resulting accuracies in the coordinates of Pluto, obtained by us (0.28" in RA, $0.31^{\prime \prime}$ in Dec.), are even better than those obtained by Barbieri (1988). This convincingly shows the fine astrometric quality of the focal field of the Baldone Schmidt telescope.

\section{The analysis of the obtained positions of Pluto}

The astrometric geocentric equatorial coordinates RA and Dec. of Pluto were calculated for the time of the observation in the FK5 system and referred to the equinox J2000.0. They are given in Table 2, together with the plate numbers taken from the journal of the observations at Baldone, calendar dates and JD of the observation. The positions of Pluto in 1991 are those from the paper by Ryl'kov et al. (1993), but as noticed above, slightly revised because of the distortion.

The obtained coordinates of Pluto were compared with the theoretical ephemeris JPL DE200. The (O-C) residuals in RA and Dec. for each exposure on plate are also given in Table 2, as well as the abbreviations of the names of the observers with the Baldone Schmidt telescope.

It has to be noted that the observing runs in 1991 and 1994 were one week long, but in 1992 and 1993 their length reached about one month. The $(\mathrm{O}-\mathrm{C})$ residuals in Dec. range from $0.55^{\prime \prime}$ to $1.5^{\prime \prime}$, about $1^{\prime \prime}$ on the average; the range of the RA residuals is given in Table 3 .

Because of the large trend in RA obtained from observations with the Normal astrograph (Ryl'kov et al. 1993) the $(\mathrm{O}-\mathrm{C})_{\mathrm{RA}}$ values are particularly interesting. These residuals for the Schmidt telescope are in sufficient agreement with those for the Normal astrograph. During the analysis of the residuals, care was taken of the fact that the positions of Pluto obtained with the Schmidt telescope are likely to contain the magnitude equation because of the large magnitude difference between the reference stars and Pluto, while the calculations for the Normal astrograph are made using secondary diffraction components of stars of 9-11 mag, which are $4.2 \mathrm{mag}$ fainter than the central images, and consequently their magnitude $(13-15)$ is almost equal to the magnitude of Pluto. Moreover, for the observations at large zenith distances the chromatic refraction assumes great significance.

As it follows from Table 2, the majority of Pluto positions differ from each other no more than $0.5^{\prime \prime}$ in RA and Dec. for each year of observations with the exception of the several plates in 1993 in RA and two plates 1991 and 1994 in Dec. Although the range of individual (O-C) residuals was rather large both in RA and Dec., no value was rejected by statistical criteria due to the next reasons.

At first, it was difficult to choose one of two quantities in the one night when the discrepancy in positions was more than $0.5^{\prime \prime}$ because these plates were remeasured and the new measurements gave practically the same results. At second, the using of the statistical criterion $3 \sigma$ to each annual period was complicated by the existence of the Pluto's satellite. The angular radius of Charon's orbit (Charon, the satellite of Pluto, was discovered in 1978, its orbital period is $\approx 6.39$ days), for a distance of $\approx 30 \mathrm{au}$, is $0.9^{\prime \prime} \pm 0.05^{\prime \prime}$ (Christy \& Harrington 1980). Recently, an improved value of $0.93^{\prime \prime}$ has been determined on the basis of 2007 observations of the Pluto-Charon system (Tholen \& Buie 1988). This value of the angular radius corresponds to the epoch 1986.5, when Pluto was at 29 au from the Earth.

One can assume that Pluto, during the period of our observations, was at a constant distance from the Earth of about $28.7 \mathrm{au}$. The angular diameter of the Charon's orbit during this period was about $1.88^{\prime \prime}$. To evaluate the position of the photometric center $x_{\mathrm{c}}$ of the Pluto-Charon system one can use the following elementary formula

$$
S_{\mathrm{PL}} x_{\mathrm{c}}=S_{\mathrm{Ch}}\left(a-x_{\mathrm{c}}\right)
$$

where $S_{\mathrm{PL}}$ and $S_{\mathrm{Ch}}$ are the areas of the Pluto's and Charon's surfaces. Taking into account that the diameter of Charon ( $\approx 600 \mathrm{~km}$ ) itself is about half of Pluto's diameter, the angular shift of the observed positions of 
Pluto (about $0.4^{\prime \prime}$ ) is the double amplitude of oscillation $\left(0.2^{\prime \prime}\right)$ as a consequence of the shift of the photometric center of the Pluto-Charon system, caused by the fact that Charon is located quite close to the planet ( $a=19.640$ $\mathrm{km}$ ), and now it is not observed as a separate object. To this constant value $0.4^{\prime \prime}$ an accidental errors of observations are added. In reality, the shift of the photometric center is a half as much because the center of mass of the system Pluto-Charon is at the angle distance about $0.11^{\prime \prime}$ from the Pluto center in the direction of Charon. If we suppose that the level of the accidental errors is no more than $0.2-0.5^{\prime \prime}$, then our estimation of the total "errors" for photographic observations of Pluto becomes $0.3-0.6^{\prime \prime}$. These values of $\sigma$ do not allow us to reject the some Pluto positions obtained by us.

All this seems to indicate that for the more exact comparison the positional observations of Pluto with its ephemerides it need to develop the formulae for the calculation of the correction for the changing of photo-center position in dependence on periodical rotation of the system. This will give the possibility to reduce the observed positions to the center mass of the system Pluto-Charon and to diminish the enough great systematic error in the positional photographic observations of Pluto.

In Table 3 the range of $(\mathrm{O}-\mathrm{C})_{\mathrm{RA}}$ values and the average $(\mathrm{O}-\mathrm{C})$ values for both coordinates with their errors are given. It is important to note that in 1994 the discrepancy between the measured coordinates of Pluto and the JPL DE200 ephemeris reaches $2.59 \pm 0.39^{\prime \prime}$ and $2.32 \pm 0.08^{\prime \prime}$ in RA for the plates of the Normal astrograph and those of the Schmidt telescope, respectively. In Dec., the situation is somewhat better because the observations are smaller than the theory by only $0.56 \pm 0.24^{\prime \prime}$ (Normal astrograph) and $0.74 \pm 0.07^{\prime \prime}$ (Baldone Schmidt telescope).

A comparison of the observations of Pluto obtained with the Schmidt telescope with the new ephemeris JPL DE202 shows that the average $(\mathrm{O}-\mathrm{C})_{\mathrm{DE}}$ residuals are $+0.58^{\prime \prime}$ and $-0.04^{\prime \prime}$ for the years 1991 and 1994, respectively. It is clear then that DE202 is a significant improvement over DE200 (see also Standish 1990a, b). The mean residuals in RA are $+0.83^{\prime \prime}$ and $+0.61^{\prime \prime}$ for 1991 and 1994 , respectively. Although they are much smaller than those derived from the comparison with DE200, they are still large enough to conclude that the theory of the DE202 ephemeris in its turn must be improved too.

Hence, to improve the numerical or to create a new analytical theory of the motion of Pluto it is necessary to continue the positional observations of the very interesting double planet system with any detector of radiation capable of registering faint (up to $B=15.7^{\mathrm{m}}$ ) images of the planet. The quality of the stellar images and the accuracy of the obtained results demonstrate that the Baldone Schmidt telescope is suitable for high-precision positional observations of stars and planets.

Acknowledgements. The authors would like to thank I. Eglitis, I. Jurgitis and I. Pundure for participating in observations with the Baldone Schmidt telescope in 1991.

\section{References}

Alksnis A., Grasberg E., Ozolina V., Platais I., 1986, In: Positional Observations of Comet Halley, Naukova Dumka, Kiev, p. 18

Alksnis A.K., 1973, in: Balklavs A.E. (ed.), The Photometrical Study of Red Stars. Zinatne Publ. House, Riga, p. 149

Andersen J., 1971, A\&A 13, 40

Barbieri C., Benacchio L., Capaccioli M., Gemmo A., 1988, AJ 96, 396

Gemmo A.G., Barbieri C., 1994, Icarus 108, 174

Christy J.W., Harrington R.S., 1980, Icarus 44, 38

Lasker B.M., Russell J.L., Jenkner H., et al., 1990, AJ 99, 2019

Murray C.A., Vectorial astrometry, Kiev, Naukova Dumka, 1986, p. 326

Murray C.A., Argyle R.A., Corben P.M., 1986, MNRAS 223, 629

Ryl'kov V.P., 1993, Kinematika Fiz. Nebesn. Tel. 6, 86

Ryl'kov V.P., Bronnikova N.M., Dement'eva A.A., Alksnis A.K., 1993, Kinematika Fiz. Nebesn. Tel. 6, 22

Standish E.M., 1990a, A\&A 233, 252

Standish E.M., 1990b, A\&A 233, 272

Steinert K.-G., On photographical astrometry with 2-m Schmidt telescope, 1987, "Current astrometry", p. 32

Tholen D.J., Buie M.W., 1988, BAAS 20, 807 